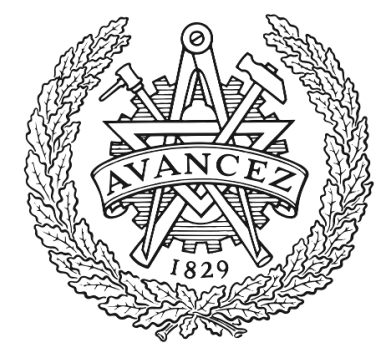

\title{
CHALMERS
}

UNIVERSITY OF TECHNOLOGY

\section{Masers: Precision probes of molecular gas}

Downloaded from: https://research.chalmers.se, 2023-04-26 10:35 UTC

Citation for the original published paper (version of record):

Richards, A., Sobolev, A., Baudry, A. et al (2020). Masers: Precision probes of molecular gas.

Advances in Space Research, 65(2): 780-789. http://dx.doi.org/10.1016/j.asr.2019.05.052

N.B. When citing this work, cite the original published paper. 


\title{
Masers: Precision probes of molecular gas
}

\author{
A.M.S. Richards ${ }^{\mathrm{a}, *}$, A. Sobolev ${ }^{\mathrm{b}}$, A. Baudry ${ }^{\mathrm{c}}$, F. Herpin ${ }^{\mathrm{c}}$, L. Decin ${ }^{\mathrm{d}}$, M.D. Gray ${ }^{\mathrm{a}}$ \\ S. Etoka ${ }^{a}$, E.M.L. Humphreys ${ }^{\mathrm{e}}$, W. Vlemmings ${ }^{\mathrm{f}}$ \\ ${ }^{a} J B C A$, School of Physics and Astronomy, University of Manchester, M13 9PL, UK \\ ${ }^{\mathrm{b}}$ Astronomical Observatory, Ural Federal University, Ekaterinburg 620083, Russia \\ ${ }^{\mathrm{c}}$ Laboratoire d'astrophysique de Bordeaux, Université de Bordeaux, 33615 Pessac, France \\ ${ }^{\mathrm{d}}$ Instituut voor Sterrenkunde, Katholieke Universiteit Leuven, 3001 Leuven, Belgium \\ ${ }^{\mathrm{e}}$ ESO, Karl-Schwarzschild-Str. 2, 85748 Garching bei Munchen, Germany \\ ${ }^{\mathrm{f}}$ Chalmers University of Technology, Onsala Space Observatory, 43992 Onsala, Sweden
}

Received 18 December 2018; received in revised form 7 May 2019; accepted 15 May 2019

Available online 11 June 2019

\begin{abstract}
Maser emission from water, methanol, silicon monoxide and other molecules can reach brightness temperatures $\gg 10^{10} \mathrm{~K}$. Such observations can achieve sub-pc precision for discs around black holes or sub-au scale interactions in protostellar discs and the regions where evolved star winds reach escape velocity. Ultra-high resolution maser observations also provide photon statistics, for fundamental physics experiments. RadioAstron has shown the success - and limitations - of cm-wave maser observations on scales $\ll 1$ mas with sparse baseline coverage. ALMA, APEX and earlier single dish searches have found a wealth of mm and sub-mm masers, some of which probably also attain high brightness temperatures. Masers are ideal for high-resolution observations throughout the radio regime and we need to consider the current lessons for the best observational strategies to meet specific science cases.
\end{abstract}

(C) 2019 COSPAR. Published by Elsevier Ltd. All rights reserved.

Keywords: Masers; Stars: AGB; Stars: supergiant; Stars: mass loss; Planetary systems: protoplanetary disks; Star formation

\section{Masers: highest spatial and velocity resolution}

The exponential nature of stimulated emission means that masers appear spectrally and spatially narrowed as well as highly amplified. Other authors in these Proceedings including Brunthaler, van Langevelde, Impellizzeri, Matthews, Moran, Kutz and Shakhvorostova have introduced evolved stars and masers and covered applications such as astrometry and extra-galactic masers. This contribution will give references for some recent Galactic maser research areas which would benefit from ultra-high resolution. We will consider the angular resolution and sensitivity

\footnotetext{
* Corresponding author.

E-mail address: amsr@jb.man.ac.uk (A.M.S. Richards).
}

required, and other benefits of observing from above the atmosphere.

Masers are one of the few natural phenomena which have spatial profiles which are usually genuinely close to Gaussian, so that fitting 2-D Gaussian components gives very accurate results, effective resolution limited by (beam size $) /(\mathrm{S} / \mathrm{N})$ where $\mathrm{S} / \mathrm{N}$ is the signal to noise ratio. This gives the beamed size of a maser spot, but if the spectral channels are much narrower than the velocity dispersion (thermal or gradient) in an emitting cloud, say $0.1 \mathrm{~km} \mathrm{~s}^{-1}$ sampling $1-$ $2 \mathrm{~km} \mathrm{~s}^{-1}$, the positions of spots in adjacent channels traces the true angular extent of the underlying cloud. This can be used to measure the beaming angle directly (Richards et al., 2011) and distinguish between emitting clumps which are approximately spherical and quiescently outflowing (which are very tightly beamed), v. shocked slabs, which can 
produce amplified emission of angular size approaching that of the emission region even in a single channel.

A list of telescope acronyms and links is given at the end.

\section{Mass loss from the stellar surface}

Asymptotic Giant Branch (AGB) and Red Supergiant (RSG) stars have diameters of 1-10 au or even greater, and surface temperatures in the range of about 2200 $3500 \mathrm{~K}$. Matthews covered observations of their radio photospheres. These stars play a major role in enriching the interstellar medium with dust and molecules containing the products of stellar nucleosynthesis. It is not wellunderstood how the wind crosses the first few $R_{\star}$ (stellar radii). These stars pulsate (with periods from a few months to several years) and the pulsations levitate the surface layers, but something else must contribute to overcoming the pull of the star's gravity. Within a few $R_{\star}$, sufficiently absorbent oxygen-rich grains would be destroyed by heating; scattering might play a role in using the stellar radiation to drive the wind but requires special grain composition (Höfner et al., 2016). It has been suggested that mass loss and dust formation might be enhanced above convection cells and that the magnetic field can shape if not drive the wind (Vlemmings et al., 2005). Outside $\sim 5 R_{\star}$, where copious dust has formed, there is ample evidence for radiation pressure on dust driving a radially accelerating wind (e.g. review by Höfner and Olofsson, 2018), reaching escape velocity a little further out. The winds are known to be clumpy (e.g. Richards et al.

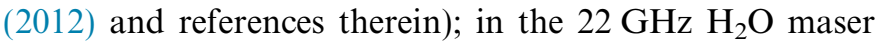
shell from $\sim 5-50 R_{\star}$ the maser clouds have a filling factor $\leq 1 \%$ but contain 10 to $>50 \%$ of the mass. The cloud size is roughly proportional to the stellar diameter, and, scaled back to the stellar surface (assuming radial expansion), is consistent with the postulated size of convection cells (Freytag et al., 2017; Chiavassa et al., 2009).
The 22-GHz maser proper motions from solitary stars are predominantly in accelerating expansion, and individual clouds appear to survive about a year ( $5 \mathrm{yr}$ - decades) around AGB (RSG), which is puzzling as they are both warmer and denser than the average of the outflow; a frozen-in magnetic field is a possible explanation. Fig. 2 shows masers observed 5 years apart. In some cases, the patterns of individual spots within a feature are recognisable, such as the inset region $\mathrm{A}$, which appears to have rotated about $60^{\circ}$ anticlockwise during 5 years. The apparent speed is about $2-15 \mathrm{~km} \mathrm{~s}^{-1}$, the uncertainty mainly due to the alignment of the epochs, but it appears to be at least partly supersonic. This could be any or all of bulk rotation, internal turbulence, or a pattern speed due to shocks/Alfvèn wave propagation. Turbulence is discussed further in Section 3.

$\mathrm{SiO}$ maser clumps, found within $\sim 5 R_{\star}$ of AGB stars, have individual motions up to $\sim 10 \mathrm{~km} \mathrm{~s}^{-1}$ but recent proper motion analysis by Assaf (2018) (using the VLBA, with a resolution of $\sim 0.04$ mas) shows that the net outflow from $\mathrm{R}$ Cas is $\sim 0.4 \mathrm{~km} \mathrm{~s}^{-1}$, taking $50-70$ yr to cross a distance of a few $R_{\star}$ (Fig. 1). This slow transport could favour dust formation. It is likely that the clumps are differentiated in density and thus temperature, degree of ionisation, magnetic and even chemical properties. These are crucial parameters in determining dust formation. ALMA provides the most precise images of precursors like AlO (e.g. Kamiński et al., 2016; Decin et al., 2017) but the relatively low brightness temperature of thermal lines makes it impractical to map them at resolutions below 10-20 mas. VLTI (e.g. Wittkowski et al., 2007; Karovicova et al., 2013) can give an indication of the distribution of the first molecules to form in a warm layer inside the surface of AGB and RSG stars (known as the MOLsphere) and the hot dust distribution but only masers can provide submas structure and kinematics as matter leaves the surface. The possibility of observing multiple transitions, along
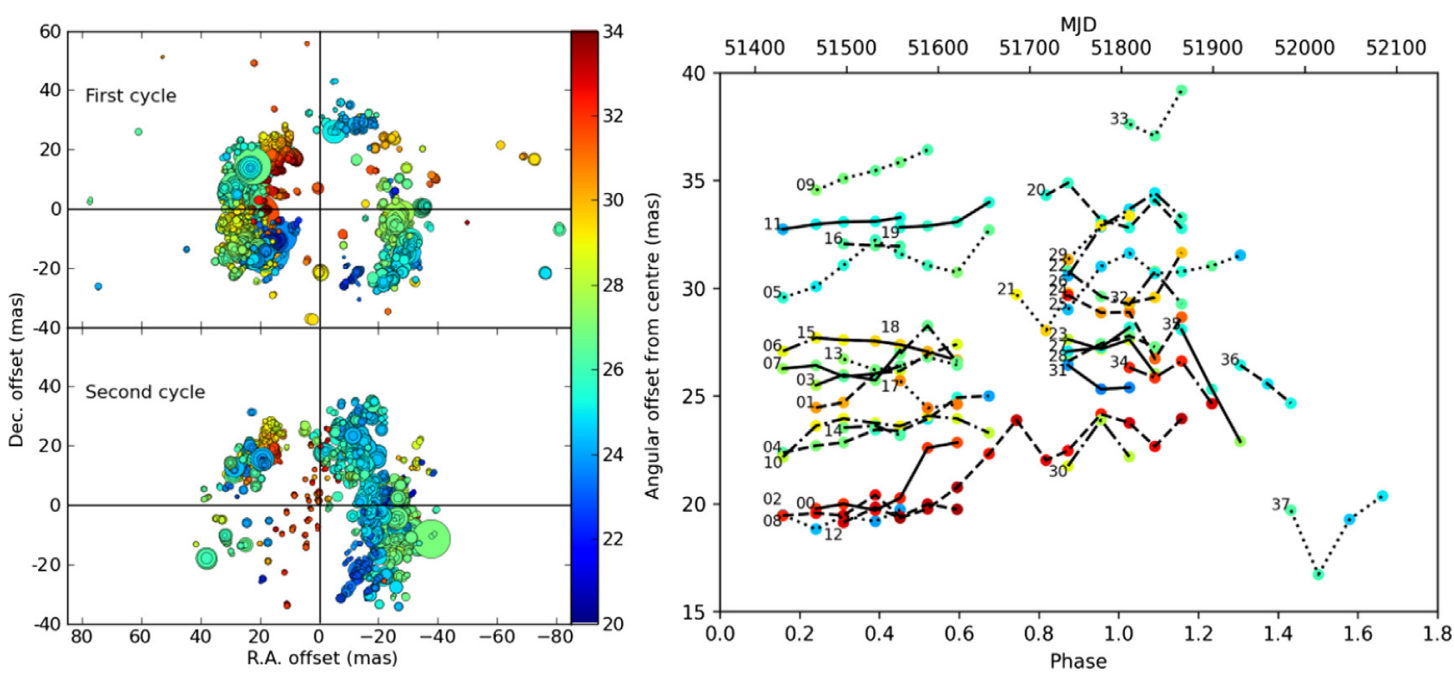

Fig. 1. left $\mathrm{R}$ Cas (at $176 \mathrm{pc}$ ): 23 epochs of $43 \mathrm{GHz} \mathrm{SiO}$ emission superimposed (each series of nearby spots in successive channels forms a feature). right The trajectories of maser features which could be matched in successive epochs as a function of stellar phase. 
with sophisticated models, now opens up the possibility of using masers to measure physical conditions as well. The masing rotational transitions of $\mathrm{SiO}$ occur at frequencies around $J_{\text {upper }} \times 43 \mathrm{GHz}$ for the upper level in $J=1-0$, $J=2-1$ etc., shifted slightly according to the isotopic composition and vibrational state; $v=1$ and $v=2$ are usually strongest but $v=0-5$ have been detected. Sub-mm $\mathrm{SiO}$ masers have been detected using APEX (De Beck and Olofsson, 2018; Humphreys et al., 2017) and mapped at $\mathrm{mm}$ wavelengths up to $J=3-2, v=1$ at $129 \mathrm{GHz}$ using the KVN (Cragg et al., 2005a). All resolved images so far show $\mathrm{SiO}$ masers are confined to the inner $\sim 7 R_{\star}$, as temperatures $>1500 \mathrm{~K}$ are required. $\mathrm{SiO}$ models and the correlations between $\mathrm{SiO}$ maser velocity, intensity and stellar pulsations (Gray et al., 2009; Wittkowski et al., 2007) show that the associated shocks are still significant, although damped in this region. Outside $\sim 5 R_{\star}$, evidence for shocks in $\mathrm{H}_{2} \mathrm{O}$ maser beaming was only seen in the thinnest-shelled Miras which also showed other evidence for flares.

Mutiple $\mathrm{mm}$ and sub-mm $\mathrm{H}_{2} \mathrm{O}$ masers have already been mapped with ALMA (Vlemmings et al., 2017; Richards et al., 2014), Fig. 3 but not yet using the full $16-\mathrm{km}$ baselines. These results broadly support the models of Neufeld and Melnick (1991), Gray et al. (2016) etc. Over $50 \mathrm{H}_{2} \mathrm{O}$ maser transitions are known or predicted between 22 and $960 \mathrm{GHz}$ in bands accessible from Earth, with more only detectable from space or at $\mathrm{THz}$ frequencies. These transitions have energy levels from 200 to $7000 \mathrm{~K}$, with pumping requirements in number density and temperature ranges $10^{5}<n<10^{11} \mathrm{~cm}^{-3}$, $300<T_{\mathrm{K}}<3000 \mathrm{~K}$. The $\mathrm{H}_{2} \mathrm{O}$ maser line at $1.296 \mathrm{THz}$ was detected from evolved stars (at a few 100 - few $1000 \mathrm{Jy}$ ) using SOFIA by Neufeld et al. (2017), along with quasi-thermal emission at $1.885 \mathrm{THz}$.
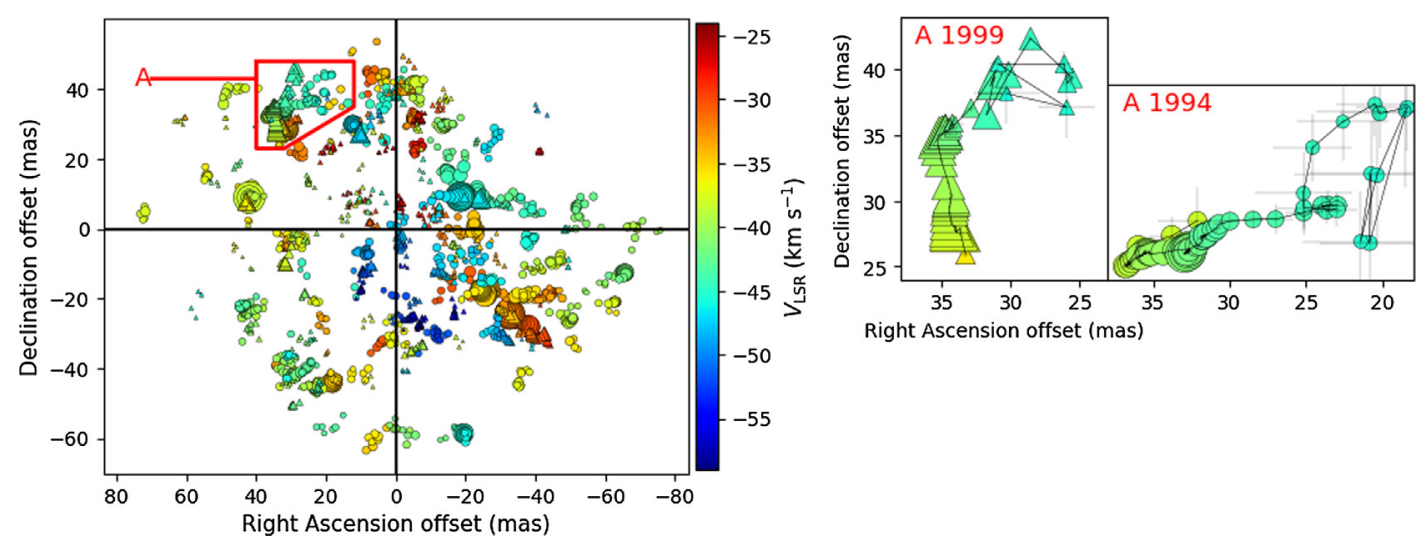

Fig. 2. left $22 \mathrm{GHz} \mathrm{H}_{2} \mathrm{O}$ masers around $\mathrm{RSG} S$ Per (2.3 kpc distant) mapped
proportional to flux density.right Separate plots for feature A at the two epochs.
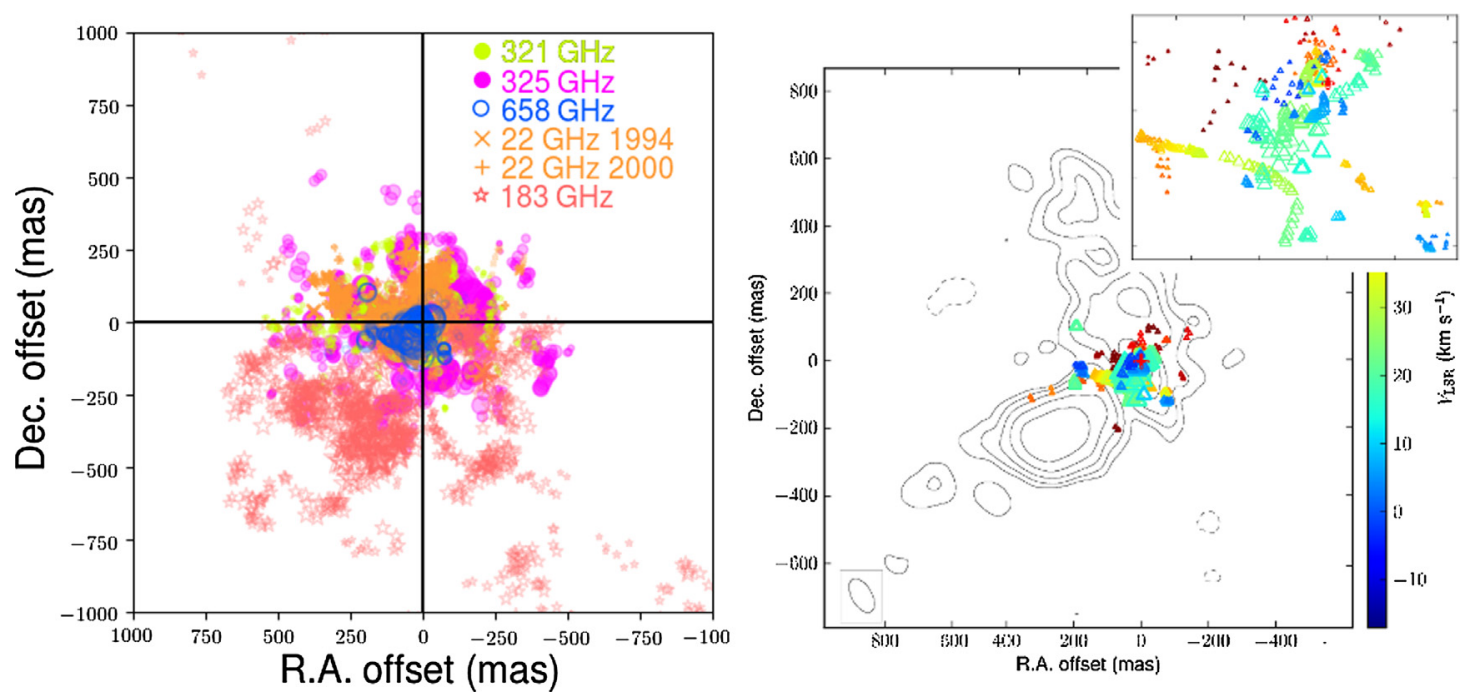

Fig. 3. left $\mathrm{H}_{2} \mathrm{O}$ masers around VY CMa (RSG, at $1.2 \mathrm{kpc}$ ) coloured by transition. right Zoom into $658 \mathrm{GHz}$ masers (coloured by velocity) and continuum (mostly dust) contours. The stellar position is marked by the red cross at $(0,0)$. (For interpretation of the references to colour in this figure legend, the reader is referred to the web version of this article.) 


\section{Star and planet formation}

Observations of methanol, mostly at 6.7 and $12 \mathrm{GHz}$, and $22 \mathrm{GHz}$ water maser emission have been performed with the EVN, VLBA and other terrestrial arrays towards many compact HII regions or Star Forming Regions (SFR) with a resolution just under 1 mas, or a few hundred $\mu$ as for $\mathrm{mm}$-wave $\mathrm{SiO}$ masers. Fitting to the Gaussian shapes of maser spots allows the astrometric accuracy to be as good as 0.006 mas (see parallax measurements for WB89437 in Hachisuka et al. (2009) using data on $\mathrm{H}_{2} \mathrm{O}$ masers, and for S252 in Reid et al. (2009) using data on methanol masers). This allows measurements of the annual trigonometric parallaxes for sources as distant as $20 \mathrm{kpc}$ from the Sun (Sanna et al., 2017). The GMVA at mm-wave line transitions (see Section 5) or Space VLBI at $22 \mathrm{GHz}$ are required to reach the highest spatial resolution currently available.

The highest current instrumental angular resolution at radio wavelengths, of about $8 \mu$ as, was achieved in observations of the extra-Galactic $\mathrm{H}_{2} \mathrm{O}$ masers in NGC 4258 (Sobolev et al., 2018; Baan et al., 2018). For maser sources in our Galaxy the best instrumental angular resolution, of about 22-23 $\mu$ as, was achieved in RadioAstron observations of $\mathrm{H}_{2} \mathrm{O}$ masers in W3 IRS 5 and W49 N (Sobolev et al., 2018; Shakhvorostova et al., 2018).

So far, the highest-resolution RadioAstron measurements of Galactic masers are from the HW3Diii region of Cepheus A. Two $\mathrm{H}_{2} \mathrm{O}$ spots were identified by Sobolev et al. (2018), with fitted sizes of $15 \mu$ as, $\leqslant 0.01$ au, corresponding to $T_{\mathrm{B}}>2 \times 10^{14} \mathrm{~K}$. These spots, separated by $0.23 \mathrm{au}$, contain $5-10 \%$ of the single dish flux, with $87 \%$ in a 0.6 au halo, see Fig. 4. Pushchino single dish monitoring shows rapid variability of the spectral feature, consistent with a very compact size. A strong IR radiation field quenches the radiative sink in $\mathrm{H}_{2} \mathrm{O}$ maser pumping (Gray et al., 2016), for example as demonstrated in the NGC 6334 I MM1 flare reported by Brogan et al. (2018), but if
IR radiation can escape from the region or the dust is cooler than the gas, very high maser amplification is possible in the dense medium over sub-au gain lengths. This could occur in the vicinity of a YSO (young stellar object) if the dust has been cleared locally and/or it is shielded by cool, optically thick dust.

The observed velocity gradient across the compact masers is equivalent to $4 \mathrm{~km} \mathrm{~s}^{-1} \mathrm{au}^{-1}$, much steeper than is seen in the ISM. The authors consider three possible scenarios producing such compact, bright masers, Keplerian motion, chance overlap of maser clouds in the line-ofsight, or turbulent vortices as a wind passes an obstacle. They consider that the first possibility is unlikely in Cep A HW3diii, while the second and third possibilities, corresponding to turbulent motions, are plausible.

It is worth noting that, as in W49, most of the maser flux is lost in Galactic $\mathrm{H}_{2} \mathrm{O}$ maser observations which only use long baselines. For example, $87 \%$ of Cep A HW3diii flux resides in a halo (Sobolev et al., 2018) whilst in W49 N only $0.1 \%$ to $0.6 \%$ of the total flux is concentrated in the ultracompact structures detected on space-ground baselines of up to 9.6 Earth diameters (Shakhvorostova et al., 2018). These low values of correlated flux density are probably due to the fact that the real sizes of the masing regions are much bigger than the apparent sizes of the spots (Gwinn, 1994; Harvey-Smith and Cohen, 2006; Sobolev et al., 2005), and the ultra-small features appear due to the turbulence and scattering either in the maser region itself or in the interstellar medium.

There have been few comprehensive searches for higherfrequency $\mathrm{H}_{2} \mathrm{O}$ masers in SFR; surveys at $183 \mathrm{GHz}$ (e.g. Cernicharo et al., 1990) found that it is widespread and often extended. ALMA imaged very bright emission in VY CMa and Sgr B2 at a few hundred mas resolution. Its excitation temperature is $200 \mathrm{~K}$, similar to $\mathrm{OH}$ masers, and it has a similar distribution around VY CMa. OH $1.6 \mathrm{GHz}$ hot spots have been detected by RadioAstron towards Onsala 1 and $\mathrm{W} 75(\mathrm{~N}) ; 183 \mathrm{GHz} \mathrm{H}_{2} \mathrm{O}$ masers share
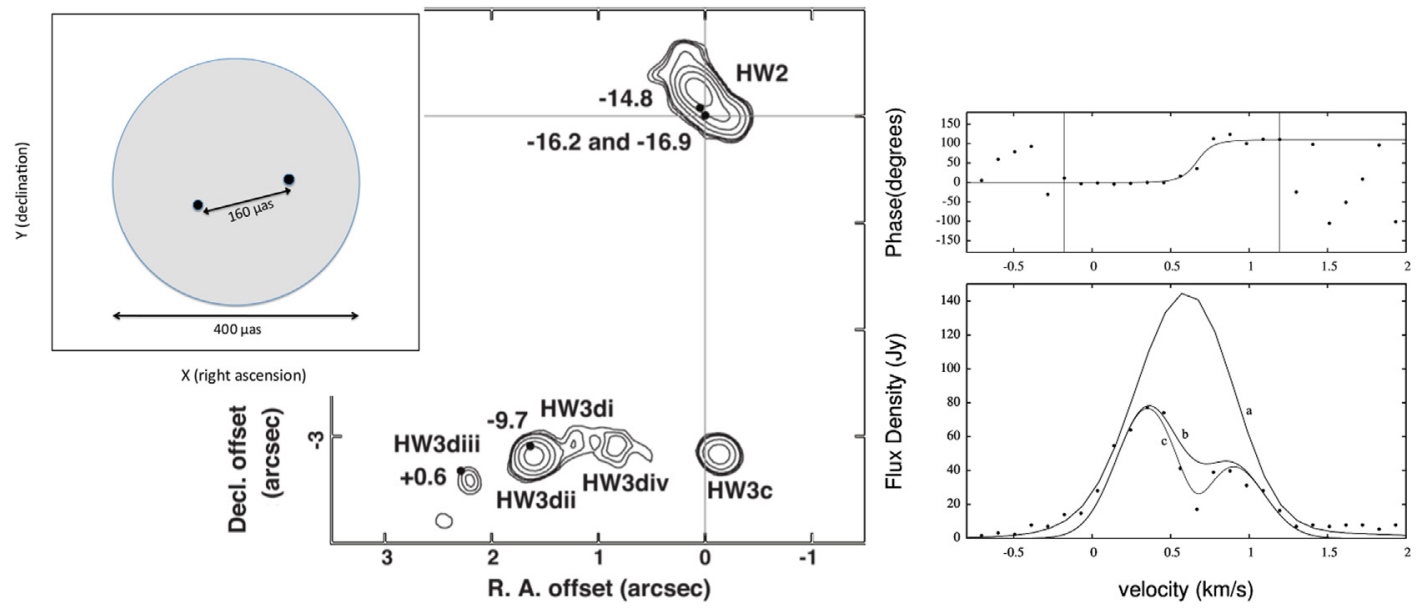

Fig. 4. centre The Cepheus A region containing HWdiii, shown in VLA 1.3-cm contours. right A ground-based baseline spectrum of the region imaged by RadioAstron. left The angular separation of the RadioAstron components, see Sobolev et al. (2018) for details. 
similar excitation conditions suggesting that they would also be detectable at sub-mas resolution. The strongest

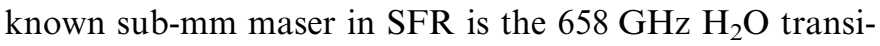
tion detected in Orion KL by Hirota et al. (2016) (along with other (sub-) mm masers). The $1.296 \mathrm{THz} \mathrm{H}_{2} \mathrm{O}$ maser was detected in NGC 7538 by Herpin et al. (2017) using SOFIA, and comparison with e-MERLIN images and Efflesburg spectra show that it comes from the hottest part of IRS1.

Numerous Class II methanol masers are found close to massive YSO (Green et al., 2017). The chemical interaction of the YSO with its environment produces a high methanol abundance and the conditions are favourable for strong maser pumping, i.e. a radiative source in the infra-red and a lower optical depth at longer wavelengths providing a sink (Ostrovskii and Sobolev, 2002). This type of pumping is supported by observations showing that excitation of these masers propagates at the speed of light (Sugiyama et al., 2008). Imaging with VLBI (e.g. Sanna et al., 2017; Araya et al., 2010; Moscadelli et al., 2017) has been used to identify outflows and infall, expanding or Keplerian discs, constraining the enclosed mass and accretion mechanisms. A maser at $390 \mathrm{GHz}$ was detected using ALMA Zinchenko et al. (2017). Fig. 5 shows two methanol transitions from the massive YSO S255 NIRS3. The line at $349.1 \mathrm{GHz}$ is both spectrally and spatially compact, likely to be the $14_{1}-14_{0} A^{-+}$Class II maser transition. The emission appears to come from the near side of the disc, at several hundred au from NIRS3, and is possibly related to this object's accretion burst in 2015. Methanol maser candidates are predicted throughout this range up to much higher frequencies (Cragg et al., 2005b).

Class I methanol masers are found at greater distances from massive YSOs, where collisional pumping takes over from the radiative source of excitation (Voronkov et al.,
2005; Sobolev et al., 2007). This is confirmed by extensive surveys e.g. Voronkov et al. (2014). These masers are numerous and widespread (Ladeyschikov et al., 2018; Yang et al., 2017). Transitions up to $44 \mathrm{GHz}$ been mapped at au resolution using KaVA (Matsumoto et al., 2014). At present Class I methanol masers are detected at frequencies up to $278 \mathrm{GHz}$ (Leurini et al., 2016) and maser candidates are predicted at much higher frequencies (Voronkov et al., 2012). Unlike Class II, they are one of the smaller range of masers also tracing lower-mass star formation.

Many class II methanol masers vary periodically (Goedhart et al., 2014), on timescales of days to months. The most probable mechanisms are variations in the infrared radiation field induced by cyclic accretion instabilities in a circumstellar (Araya et al., 2010) or protobinary (Parfenov and Sobolev, 2014) disc; alternative possibilities include variability of a central YSO (Inayoshi et al., 2013) or a background source providing seed photons. Where regions have multiple features at different velocities, or additional masers e.g. $\mathrm{HCHO}, \mathrm{H}_{2} \mathrm{O}$, high resolution imaging can locate the variable features, so that their orientation in relation to time lags reveals the speed of propagation and thus the nature of the excitation (Moscadelli et al., 2017; Szymczak et al., 2016; Sugiyama et al., 2008). The $6.7 \mathrm{GHz}$ methanol maser emission flares from S255 NIRS3 (Szymczak et al., 2018) appear to be connected to accretion bursts which could provide a solution to the radiative pressure limiting the accretion of matter onto the YSO allowing it to grow in mass beyond $\sim 10$ $\mathbf{M}_{\odot}$. Similar flares are seen in NGC6334 1 (Brogan et al., 2018). Discs around massive YSOs are subject to gravitational instability (Meyer et al., 2019), likely to lead to frequent accretion bursts, a suggestion supported by the structures observed in maser emission (Sanna et al., 2017; Zinchenko et al., 2017).
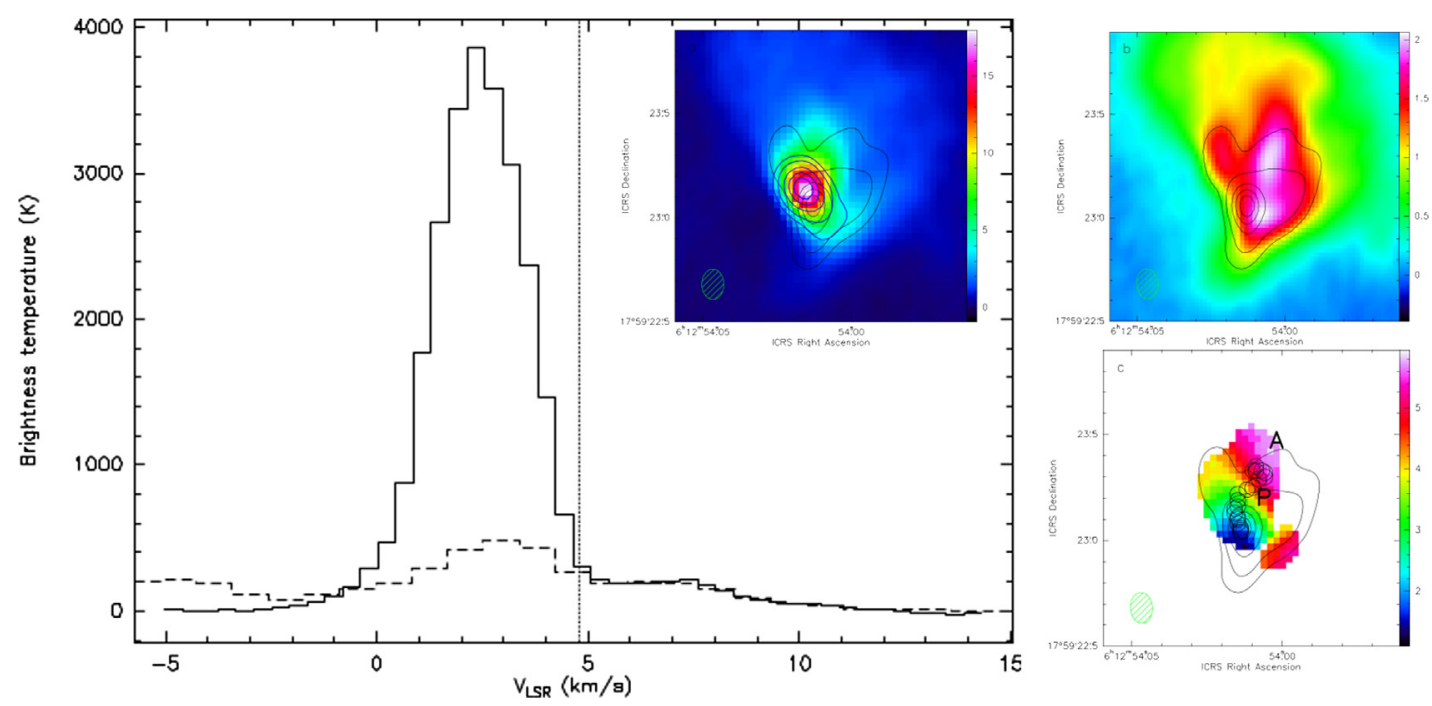

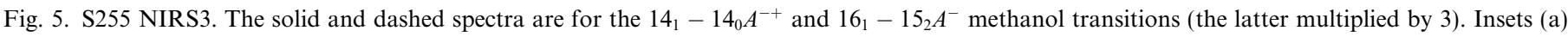

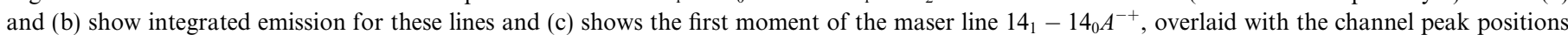

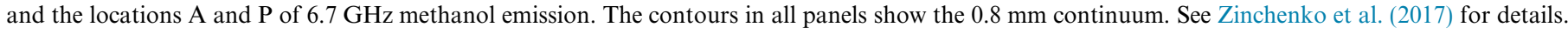


The remote heating mechanism is supported by an investigation of beaming by Araya et al. (2010), who found an inverse relationship between the measured (beamed) size and the flux density of maser spots. This suggests an "amplification bounded" maser in which the emission is from clouds which are very roughly spherical and probably drifting quiescently, rather than shocked slabs (see Section 1).

\section{Maser and fundamental physics}

A change by a factor of a few or even a few tenths in the maser pump rate or amplification column can lead to orders of magnitude increases in brightness (introduced by e.g. Elitzur, 1992 and Gray et al., 2016). Maser flares have been observed sporadically and periodically, and understanding the maser mechanism reveals the underlying process. There are at least three ways to produce a flare. Two clouds overlapping along the line of sight has been directly observed in W Hya (Richards et al., 2012) by tracing the proper motions of features, one clump passing in front of another with a similar $V_{\mathrm{LSR}}$, whilst the associated spectral peak rose from $\sim 10$ to nearly $3000 \mathrm{Jy}$. This process, and the effects of cloud shape, are modelled by Gray et al. (2018). Shocks can also produce flares, and one resolved example may occur close to VY CMa. The curve of $658 \mathrm{GHz}$ masers in the inset of Fig. 2 appears to represent wind deflected by the large SE dust clump. In 2013-2016 a new 22-GHz maser feature appeared, brightening to $>2000 \mathrm{Jy}$ just behind this region, and a $\sim 1000$ Jy peak is seen nearby at $183 \mathrm{GHz}$ in 2016 observations. In both cases the peak is around $-1 \mathrm{~km} \mathrm{~s}^{-1}$, blue-shifted compared with the normal peaks around $V_{\star}, 22 \mathrm{~km} \mathrm{~s}^{-1}$. However, more precise astrometry and higher resolution than the existing $\mathrm{mm}$ images are needed to investigate this properly. Thirdly, IR emission can enhance the pump rate by heating the gas. This is seen in the variations of circumstellar $\mathrm{SiO}$ (also affected by pulsations), and the weak variability of $\mathrm{H}_{2} \mathrm{O}$ masers with stellar period. High resolution is needed to locate the maser species, along with monitoring any time delays between events in different locations. This distinguishes between the timescale needed for propagation of shocks, or radiative effects which would affect a large area more rapidly.

Precise tests of turbulence models can be made using ultra-high resolution mapping, whether by direct observation of small regions (Sobolev et al., 2018, see Section 3) or by consideration of the maser images, profiles and variability (Liljeström and Gwinn, 2000; Sobolev et al., 1998, 2003). The scale at which turbulence is initiated in a region can be investigated by considering maser distribution statistics (Gwinn, 1994; Sobolev et al., 1998, 2003). Fractal analysis of clustering scales in angular separation (including larger scales) and velocity provided a measure of the Kolmogorov scale, distinguishing between incompressible and compressible flows, the latter suggesting supersonic dissipation (Gray, 2012; Strelnitski et al., 2002; Silant'ev et al., 2006; Richards et al., 2005). Probing the scales on which turbulence is dissipated requires very high angular resolution and was probably first measured using space VLBI with RadioAstron (Sobolev et al., 2018).

The extreme brightness of masers even allows high enough resolution to investigate non-Gaussian statistics (Gray, 2012), as saturated masers may show residual coherence due to the stimulated emission favouring the fundamental (Lorenzian) line shape (of order $2 \mathrm{~Hz}$ at $22 \mathrm{GHz}$ ). Takefuji et al. (2016) used rapid ( $\mu$ s) time sampling at ultra-high spectral resolution with a single dish to find a coherence scale of $\sim 30 \mu$ s for bright masers in W49N, 6 times longer than expected for Gaussian statistics. We note that the measured coherence times vary between sources observed, and also across the spectral bandwidth of each individual source, making the coherence detections unlikely to be an artefact of the observational set-up. However, further tests at high spatial resolution are needed to provide robust tests of this effect. It might be easier to sample higher frequencies where an intrinsically broader line allows slower sampling, e.g. $183 \mathrm{GHz}$, but ideally, one would use high spatial resolution also to ensure individual maser features are isolated, and there may be problems with the timescale of phase calibration for ground-based observations. However, more conventional interferometry to identify isolated features could complement high timeand frequency-resolution space monitoring.

\section{Potential and practical considerations of space VLBI}

In modelling physical conditions from multiple transitions, the most accurate results require detecting as much as possible of the emission from each transition, at sufficient resolution to isolate individual clouds or regions of approximately homogenous conditions and determine whether the different masers co-propagate or are separate. Measuring the beaming angle (and using this as a diagnostic for the presence of shocks) also requires detecting the full extent of the parent clumps.

Although the brightest masers are often beamed into sub-mas spots, with brightness temperatures exceeding $10^{12} \mathrm{~K}$, these represent the most favourable velocity gradients along long path lengths, e.g. the centre of a cloud. Emission from the edges of a cloud, or from shocks, can be as extended as the emitting region, tens or even hundreds of mas from Galactic sources. It is well-known that current VLBI arrays with very sparse/no coverage on baselines less than a few $100 \mathrm{~km}$ resolve-out up to $90 \%$ of even $\mathrm{SiO}$ maser emission from evolved stars within a few $\mathrm{kpc}$. This is less severe for star-forming regions, e.g. Issaoun et al. (2017) recovered $1 / 3$ of $3-\mathrm{mm} \mathrm{SiO}$ emission from a protostar in Orion.

e-MERLIN (+ EVN at 4-6 GHz) and ALMA currently provide a suitable range of angular scales in their respective frequency regimes. In $1 \mathrm{~h}$ or less, ALMA could detect submm masers of $T_{\mathrm{B}} \leq 3 \times 10^{4} \mathrm{~K}$ (enough to be sure the emission is non-thermal) at 5-20 mas resolution, providing the 
required sub-mas accuracy through component fitting. At $\mathrm{mm}$ wavelengths, LLAMA and/or the long-term plan for ALMA extension to $20-50 \mathrm{~km}$ are required. In addition, these arrays provide the shorter spacings and sensitivity to detect AGB (RSG) within a few $100 \mathrm{pc}(\mathrm{kpc})$ at $22 \mathrm{GHz}$ and above. The current gap at $43 \mathrm{GHz}$ between VLA and VLBI resolution will be filled by the ngVLA.

The highest resolution maser observations to date have been made with the GMVA at $86 \mathrm{GHz}$ and RadioAstron at $22 \mathrm{GHz}$. Whilst circumstellar $86 \mathrm{GHz} \mathrm{SiO}$ masers have been readily detected, the only $22 \mathrm{GHz}$ masers detected at a comparable $\sim 10-20 \mu$ as resolution have been from starforming regions and extra-galactic sources. The next steps in determining what (if any) $\mathrm{H}_{2} \mathrm{O}$ and other masers are detectable at similar or higher resolution will be taken, firstly, when $230-325 \mathrm{GHz}$ or higher frequency spectral line VLBI with ALMA is offered and secondly, by adding more shorter spacings to RadioAstron data which may provide sufficient constraints to image $22 \mathrm{GHz}$ hot spots around evolved stars. The RadioAstron Cep A results also point to SFR as good targets for $\mathrm{mm} / \mathrm{sub}-\mathrm{mm}_{2} \mathrm{O}$ masers with ground-based and space VLBI.

Thus, some of the applications of space VLBI require a range of ground-based spacings. This implies that if e.g. ALMA or the SKA are being phased-up, some antennas should remain on their long baselines. In fact, it is often desirable to sample maser variability on daily - monthly time scales. It is most efficient to use frequent single-dish monitoring, which can be used to track changes between more time-consuming interferometry sessions, probably with restricted availability for practical reasons.

It is not unlikely that some $\mathrm{mm}$ and sub-mm maser have the required $T_{\mathrm{B}} \geqslant 10^{11} \mathrm{~K}$ to be detected, for example, on baselines 2.5 Earth diameters in 5-10 min. Mapping maser hot-spots alone will reveal cicumstellar kinematics on scales $<R_{\star}$ around evolved stars. Separate, complementary ALMA, VLTI etc. observations will reveal linked variations in abundance of dust precursors and nascent dust. Mapping $\mathrm{H}_{2} \mathrm{O}$ masers on sub-au scales in protoplanetary discs is a very exciting prospect, as not only could they trace the dynamics of gas flow, but also the distribution of water.

Some of the brightest known $\mathrm{H}_{2} \mathrm{O}$ masers from lower energy states, e.g. 183 and $325 \mathrm{GHz}$, are highly absorbed from most terrestrial sites, making VLBI difficult except for ALMA and SPT. These trace cooler and more extended regions where thermal lines have low surface brightness, and so masers are the only means of imaging such regions at high resolution. Other predicted maser lines $(620,793$, $899,1077,1486,1689,1849,1873 \mathrm{GHz})$ are completely unobservable from Earth; the Kuiper Airborne Observatory, Herschel and SOFIA have detected some but at spectral resolution too low to exploit their maser nature. Spacespace VLBI - or even single-dish monitoring from space at high spectral resolution - will be especially beneficial for these transitions. Space VLBI offers the exciting possibility of making annual parallax measurements for extragalactic sources. The required angular resolution is already possible (Sobolev et al., 2018), but phase referencing is challenging and space-space VLBI (no atmospheric delay) might be more practical.

In conclusion, space VLBI observations of masers at wavelengths from $\mathrm{cm}$ to sub-mm will probe the layers around evolved stars where mass loss is initiated and contribute to a better understanding of the mass loss mechanism. The observations will help to unravel the accretion process of massive stars and possibly the sub-au scale dynamics of water in protoplanetary discs. Ultra-high resolution in time, space and frequency will reach into the fundamental physics of spectral line formation.

\section{Acknowledgement}

AMSR is employed by the UK ALMA Regional Centre, supported by STFC. AMS was supported by the Russian Science Foundation (grant 18-12-00193). We thank the referees for very helpful comments which have enriched this paper.

\section{Appendix A. Telescope acronyms and links}

ALMA: Atacama Large sub-millimeter and Millimeter Array http://www.almascience.org.

APEX: Atacama Pathfinder Experiment http://www. apex-telescope.org.

Efflesberg Radio Telescope https://www.mpifr-bonn. mpg.de/en/effelsberg.

EVN: European VLBI Network https://www.evlbi.org.

GMVA: Global Millimeter VLBI Array https://www3. mpifr-bonn.mpg.de/div/vlbi/globalmm.

Kuiper Airborne Observatory http://quest.arc.nasa. gov/lfs/about-kao.html.

Herschel http://sci.esa.int/herschel.

KaVA: KVN and VERA Array https://radio.kasi.re.kr/ kava/index.php KVN: Korean VLBI Network https://radio.kasi.re.kr/kvn/main_kvn.php.

e-MERLIN: http://www.e-MERLIN.ac.uk.

LLAMA: Large Latin American Millimeter Array https://www.llamaobservatory.org.

Pushchino Radio Astronomy Observatory http://www. prao.ru/English.

RadioAstron http://www.asc.rssi.ru/radioastron.

SOFIA: Stratospheric Observatory for Infrared Astronomy https://www.sofia.usra.edu.

SPT: South Pole Telescope https://pole.uchicago.edu/ spt.

VERA: VLBI Exploration of Radio Astrometry http:// veraserver.mtk.nao.ac.jp VLBA: Very Long Baseline Array https://public.nrao.edu/telescopes/vlba.

VLA: Very Long Array https://public.nrao.edu/telescopes/vla.

VLTI: Very Large Telescope Interferometry https:// www.eso.org/sci/facilities/paranal/telescopes/vlti.html. 


\section{References}

Araya, E.D., Hofner, P., Goss, W.M., Kurtz, S., Richards, A.M.S., Linz, H., Olmi, L., Sewiło, M., 2010. Quasi-periodic formaldehyde maser flares in the massive protostellar object IRAS 18566+0408. Astrophys. J. Lett. 717, L133-L137. https://doi.org/10.1088/2041-8205/717/2/ L133 1006.2164.

Assaf, K.A., 2018. Multi-epoch proper motion magnetic field comparison of $\mathrm{SiO}$ masers around R cas. Astrophys. J. 869, 80. https://doi.org/ 10.3847/1538-4357/aaea65.

Baan, W., Alakoz, A., An, T., Ellingsen, S., Henkel, C., Imai, H., Kostenko, V., Litovchenko, I., Moran, J., Sobolev, A., Tolmachev, A., 2018. $\mathrm{H}_{2} \mathrm{O}$ MegaMasers: radioAstron success story. In: Tarchi, A., Reid, M.J., Castangia, P. (Eds.), Astrophysical Masers: Unlocking the Mysteries of the Universe, volume 336 of IAU Symposium, Cambridge University Press, Cambridge, UK, pp. 422-425. doi: https://doi.org/ 10.1017/S1743921317010869. arXiv: 1801.08796.

Araya, E.D., Hofner, P., Goss, W.M., Kurtz, S., Richards, A.M.S., Linz, H., Olmi, L., Sewiło, M., 2010. Quasi-periodic Formaldehyde maser flares in the massive protostellar object IRAS 18566+0408. Astrophys. J. Lett. 717, L133-L137. https://doi.org/10.1088/2041-8205/717/2/ L133 1601.03197.

Brogan, C.L., Hunter, T.R., Cyganowski, C.J., Chibueze, J.O., Friesen, R. K., Hirota, T., MacLeod, G.C., McGuire, B.A., Sobolev, A.M., 2018. The extraordinary outburst in the massive protostellar system NGC 6334I-MM1: flaring of the water masers in a North-South bipolar outflow driven by MM1B. Astrophys. J. Lett. 866, 87. https://doi.org/ 10.3847/1538-4357/aae151 1809.04178.

Cernicharo, J., Thum, C., Hein, H., John, D., Garcia, P., Mattioco, F., 1990. Detection of $183 \mathrm{GHz}$ water vapor maser emission from interstellar and circumstellar sources. A\&A 231, L15-L18.

Chiavassa, A., Plez, B., Josselin, E., Freytag, B., 2009. Radiative hydrodynamics simulations of red supergiant stars. I. Interpretation of interferometric observations. A\&A 506, 1351-1365. https://doi.org/ 10.1051/0004-6361/200911780 0907.1860.

Cho, S.-H., Yun, Y., Kim, J., Yoon, D.-H., Kim, D.-J., Choi, Y.K., Dodson, R., Rioja, M., Imai, H., A study on evolved stars by simultaneous observations of $\mathrm{H}_{2} \mathrm{O}$ and $\mathrm{SiO}$ masers using $\mathrm{KVN}$. In: Tarchi, A., Reid, M.J., Castangia, P. (Eds.), Astrophysical Masers: Unlocking the Mysteries of the Universe, volume 336 of IAU Symposium, Cambridge University Press, Cambridge, UK, 2018, pp. 359-364. doi: 10.1017/S1743921317010274.

Cragg, D.M., Sobolev, A.M., Godfrey, P.D., 2005b. Models of class II methanol masers based on improved molecular data. MNRAS 360, 533-545. https://doi.org/10.1111/j.1365-2966.2005.09077.x, arXiv: astro-ph/0504194.

De Beck, E., Olofsson, H., 2018. Circumstellar environment of the M-type AGB star R Doradus. APEX spectral scan at 159.0-368.5 GHz. A\&A 615, A8. https://doi.org/10.1051/0004-6361/201732470, arXiv:1801.07984.

Decin, L., Richards, A.M.S., Waters, L.B.F.M., Danilovich, T., Gobrecht, D., Khouri, T., Homan, W., Bakker, J.M., Van de Sande, M., Nuth, J.A., De Beck, E., 2017. Study of the aluminium content in AGB winds using ALMA. Indications for the presence of gas-phase $\left(\mathrm{Al}_{2} \mathrm{O}_{3}\right)_{n}$ clusters, A\&A 608, A55. doi: https://doi.org/10.1051/00046361/201730782. arXiv: 1704.05237.

Elitzur, M., 1992. Astronomical Masers, Kluwer, Dordrecht.

Freytag, B., Liljegren, S., Höfner, S., 2017. Global 3D radiationhydrodynamics models of AGB stars. Effects of convection and radial pulsations on atmospheric structures. A\&A 600, A137. https://doi.org/ 10.1051/0004-6361/201629594, arXiv:1702.05433.

Goedhart, S., Maswanganye, J.P., Gaylard, M.J., van der Walt, D.J., 2014. Periodicity in Class II methanol masers in high-mass starforming regions. MNRAS 437, 1808-1820. https://doi.org/10.1093/ mnras/stt2009 1311.1615.

Gray, M.D., Wittkowski, M., Scholz, M., Humphreys, E.M.L., Ohnaka, K., Boboltz, D., 2009. Sio maser emission in miras. MNRAS 394, 5166. https://doi.org/10.1111/j.1365-2966.2008.14237.x 0811.2770.
Gray, M.D., Baudry, A., Richards, A.M.S., Humphreys, E.M.L., Sobolev, A.M., Yates, J.A., 2016. The physics of water masers observable with ALMA and SOFIA: model predictions for evolved stars. MNRAS 456, 374-404. https://doi.org/10.1093/mnras/stv2437, arXiv: 1510.06182 .

Gray, M.D., Mason, L., Etoka, S., 2018. A new 3D maser code applied to flaring events. MNRAS 477, 2628-2639. https://doi.org/10.1093/mnras/sty576, arXiv:1803.01625.

Gray, M., 2012. Maser Sources in Astrophysics. Cambridge University Press, Cambridge, UK.

Green, J.A., Breen, S.L., Fuller, G.A., McClure-Griffiths, N.M., Ellingsen, S.P., Voronkov, M.A., Avison, A., Brooks, K., Burton, M.G., Chrysostomou, A., Cox, J., Diamond, P.J., Gray, M.D., Hoare, M.G., Masheder, M.R.W., Pestalozzi, M., Phillips, C., Quinn, L.J., Richards, A.M.S., Thompson, M.A., Walsh, A.J., Ward-Thompson, D., Wong-McSweeney, D., Yates, J.A., 2017. The 6-GHz multibeam maser survey - II. Statistical analysis and Galactic distribution of 6668MHz methanol masers, MNRAS 469, 1383-1402. doi: https://doi.org/ $10.1093 / \mathrm{mnras} / \mathrm{stx} 887$.

Gwinn, C.R., 1994. Scattered halos around $\mathrm{H}_{2} \mathrm{O}$ masers. Astrophys. J. Lett. 431, L123-L126. https://doi.org/10.1086/187488.

Hachisuka, K., Brunthaler, A., Menten, K.M., Reid, M.J., Hagiwara, Y., Mochizuki, N., 2009. The distance to a star-forming region in the outer arm of the galaxy. Astrophys. J 696, 1981-1986. https://doi.org/ 10.1088/0004-637X/696/2/1981 0901.2481.

Harvey-Smith, L., Cohen, R.J., 2006. Discovery of large-scale methanol and hydroxyl maser filaments in W3(OH). MNRAS 371, 1550-1558. https://doi.org/10.1111/j.1365-2966.2006.10806.x.

Herpin, F., Baudry, A., Richards, A.M.S., Gray, M.D., Schneider, N., Menten, K.M., Wyrowski, F., Bontemps, S., Simon, R., Wiesemeyer, H., 2017. First detection of a THz water maser in NGC 7538-IRS1 with SOFIA and new $22 \mathrm{GHz}$ e-MERLIN maps. A\&A 606, A52. https://doi.org/10.1051/0004-6361/201731056, arXiv:1706.06390.

Hirota, T., Machida, M.N., Matsushita, Y., Motogi, K., Matsumoto, N., Kim, M.K., Burns, R.A., Honma, M., 2016. ALMA band 8 continuum emission from orion source I. Astrophys. J. 833, 238. https://doi.org/10.3847/1538-4357/833/2/238, arXiv:1611.00855.

Humphreys, E.M.L., Immer, K., Gray, M.D., De Beck, E., Vlemmings, W.H.T., Baudry, A., Richards, A.M.S., Wittkowski, M., Torstensson, K., De Breuck, C., Møller, P., Etoka, S., Olberg, M., 2017. Simultaneous $183 \mathrm{GHz} \mathrm{H}_{2} \mathrm{O}$ maser and $\mathrm{SiO}$ observations towards evolved stars using APEX SEPIA Band 5. A\&A 603, A77. https://doi. org/10.1051/0004-6361/201730718, arXiv:1704.02133.

Höfner, S., Olofsson, H., 2018. Mass loss of stars on the asymptotic giant branch. Mechanisms, models and measurements. A\&ARev 26, 1. https://doi.org/10.1007/s00159-017-0106-5.

Höfner, S., Bladh, S., Aringer, B., Ahuja, R., 2016. Dynamic atmospheres and winds of cool luminous giants. I. $\mathrm{Al}_{2} \mathrm{O}_{3}$ and silicate dust in the close vicinity of M-type AGB stars, A\&A 594, A108. doi: https://doi. org/10.1051/0004-6361/201628424. arXiv: 1605.09730.

Inayoshi, K., Sugiyama, K., Hosokawa, T., Motogi, K., Tanaka, K.E.I., 2013. Direct diagnostics of forming massive stars: stellar pulsation and periodic variability of maser sources. Astrophys. J. Lett. 769, L20. https://doi.org/10.1088/2041-8205/769/2/L20 1304.5241.

Issaoun, S., Goddi, C., Matthews, L.D., Greenhill, L.J., Gray, M.D., Humphreys, E.M.L., Chandler, C.J., Krumholz, M., Falcke, H., 2017. VLBA imaging of the $3 \mathrm{~mm} \mathrm{SiO}$ maser emission in the disk-wind from the massive protostellar system Orion Source I. A\&A 606, A126. https://doi.org/10.1051/0004-6361/201731548, arXiv:1707.07455.

Kamiński, T., Wong, K.T., Schmidt, M.R., Müller, H.S.P., Gottlieb, C. A., Cherchneff, I., Menten, K.M., Keller, D., Brünken, S., Winters, J. M., Patel, N.A., 2016. An observational study of dust nucleation in Mira (o Ceti). I. Variable features of $\mathrm{AlO}$ and other Al-bearing species, A\&A 592, A42. doi: https://doi.org/10.1051/0004-6361/201628664. arXiv: 1604.05641.

Karovicova, I., Wittkowski, M., Ohnaka, K., Boboltz, D.A., Fossat, E., Scholz, M., 2013. New insights into the dust formation of oxygen-rich 
AGB stars. A\&A 560, A75. https://doi.org/10.1051/0004-6361/ 2013223761310.1924.

Ladeyschikov, D.A., Bayandina, O.S., Sobolev, A.M., 2018. The Class I Methanol Masers Database: Status and Prospects. In: Modern Star Astronomy. Vol. 1, Astronomy-2018 (XIII Congress of the International Public Organization "Astronomical Society"). Conference Abstracts, Moscow: IZMIRAN, 2018. p. 208-211, volume 1, Cambridge Scientific Publishers, Cambridge, UK, 2018, pp. 208-211. doi: https://doi.org/10.31361/eaas.2018-1.045.

Leurini, S., Menten, K.M., Walmsley, C.M., 2016. Physical characteristics of bright Class I methanol masers. A\&A 592, A31. https://doi.org/ 10.1051/0004-6361/201527974, arXiv:1605.09406.

Liljeström, T., Gwinn, C.R., 2000. Water masers diagnosing postshocked conditions in W49N. Astrophys. J. 534, 781-800. https://doi.org/ 10.1086/308781 astro-ph/0002299.

Matsumoto, N., Hirota, T., Sugiyama, K., Kim, K.-T., Kim, M., Byun, D.-Y., Jung, T., Chibueze, J.O., Honma, M., Kameya, O., Kim, J., Lyo, A.-R., Motogi, K., Oh, C., Shino, N., Sunada, K., Bae, J., Chung, H., Chung, M.-H., Cho, S.-H., Han, M.-H., Han, S.-T., Hwang, J.-W., Je, D.-H., Jike, T., Jung, D.-K., Jung, J.-s., Kang, J.-h., Kang, J., Kang, Y.-W., Kan-ya, Y., Kawaguchi, N., Kim, B.G., Kim, J., Ryoung Kim, H., Kim, H.-G., Kobayashi, H., Kono, Y., Kurayama, T., Lee, C., Lee, J.A., Lee, J., Lee, J.-W., Lee, S.H., Lee, S.-S., Minh, Y.C., Miyazaki, A., Oh, S.-J., Oyama, T., Park, S.-y., Roh, D.-G., Sasao, T., Sawada-Satoh, S., Shibata, K.M., Sohn, B.W., Song, M.-G., Tamura, Y., Wajima, K., Wi, S.-O., Yeom, J.-H., Yun, Y.J., 2014. The First Very Long Baseline Interferometry Image of a 44 $\mathrm{GHz}$ Methanol Maser with the KVN and VERA Array (KaVA), Astrophys. J. Lett. 789, L1. doi:https://doi.org/10.1088/2041-8205/ 789/1/L1. arXiv:1406.2086.

Meyer, D.M.-A.,Vorobyov, E.I., Elbakyan, V.G., Stecklum, B., Eislöffel, J., Sobolev, A.M., 2019. Burst occurrence in young massive stellar objects. MNRAS 482, 5459-5476. doi:https://doi.org/10.1093/mnras/ sty2980. arXiv:1811.00574.

Moscadelli, L., Sanna, A., Goddi, C., Walmsley, M.C., Cesaroni, R., Caratti o Garatti, A., Stecklum, B., Menten, K.M., Kraus, A., 2017. Extended $\mathrm{CH}_{3} \mathrm{OH}$ maser flare excited by a bursting massive YSO, A\&A 600, L8. doi: https://doi.org/10.1051/0004-6361/201730659.

Neufeld, D.A., Melnick, G.J., 1991. Excitation of millimeter and submillimeter water masers. Astrophys. J. 368, 215-230.

Neufeld, D.A., Melnick, G.J., Kaufman, M.J., Wiesemeyer, H., Güsten, R., Kraus, A., Menten, K.M., Ricken, O., Faure, A., 2017. SOFIA/ GREAT discovery of terahertz water masers. Astrophys. J. 843, 94. https://doi.org/10.3847/1538-4357/aa7568, arXiv:1705.09672.

Ostrovskii, A.B., Sobolev, A.M., 2002. Influence of dust parameters on class II methanol maser pumping. In: Migenes, V., Reid, M.J. (Eds.), Cosmic Masers: From Proto-Stars to Black Holes, volume 206 of IAU Symposium. Astronomical Society of the Pacific, San Francisco, USA, pp. 183-186.

Parfenov, S.Y., Sobolev, A.M., 2014. On the Class II methanol maser periodic variability due to the rotating spiral shocks in the gaps of discs around young binary stars. MNRAS 444, 620-628. https://doi.org/ 10.1093/mnras/stu1481 1407.7708.

Reid, M.J., Menten, K.M., Brunthaler, A., Zheng, X.W., Moscadelli, L., $\mathrm{Xu}$, Y., 2009. Trigonometric parallaxes of massive star-forming regions. I.S $252 \&$ G232.6+1.0, Astrophys. J. 693, 397-405. doi: https://doi.org/10.1088/0004-637X/693/1/397. arXiv: 0811.0595.

Richards, A.M.S., Cohen, R.J., Crocker, M., Lekht, E.E., Mendoza, E., Samodurov, V.A., 2005. Merlin and Puschino Observations of $\mathrm{H}_{2} \mathrm{O}$ Masers in Outer Galactic SFR S128N. AP\&SS 295, 19-25. https://doi. org/10.1007/s10509-005-3652-7.

Richards, A.M.S., Elitzur, M., Yates, J.A., 2011. Observational evidence for the shrinking of bright maser spots. A\&A 525, A56 1010.4419.

Richards, A.M.S., Etoka, S., Gray, M.D., Lekht, E.E., Mendoza-Torres, J.E., Murakawa, K., Rudnitskij, G., Yates, J.A., 2012. Evolved star water maser cloud size determined by star size. A\&A 546, A16. https:// doi.org/10.1051/0004-6361/201219514 1207.2583.
Richards, A.M.S., Impellizzeri, C.M.V., Humphreys, E.M., Vlahakis, C., Vlemmings, W., Baudry, A., De Beck, E., Decin, L., Etoka, S., Gray, M.D., Harper, G.M., Hunter, T.R., Kervella, P., Kerschbaum, F., McDonald, I., Melnick, G., Muller, S., Neufeld, D., O’Gorman, E., Parfenov, S.Y., Peck, A.B., Shinnaga, H., Sobolev, A.M., Testi, L., Uscanga, L., Wootten, A., Yates, J.A., Zijlstra, A., 2014. ALMA sub$\mathrm{mm}$ maser and dust distribution of VY Canis Majoris. A\&A 572, L9. https://doi.org/10.1051/0004-6361/201425024 1409.5497.

Sanna, A., Reid, M.J., Dame, T.M., Menten, K.M., Brunthaler, A., 2017. Mapping spiral structure on the far side of the Milky Way, Science 358 , 227-230. doi: https://doi.org/10.1126/science.aan5452. arXiv:1710.06489.

Shakhvorostova, N.N., Alakoz, A.V., Sobolev, A.M., 2018. Brightness temperatures of galactic masers observed in the RadioAstron project, In: Tarchi, A., Reid, M.J., Castangia, P. (Eds.), Astrophysical Masers: Unlocking the Mysteries of the Universe, volume 336 of IAU Symposium, Cambridge University Press, Cambridge, UK, 2018, pp. 447-448. doi: https://doi.org/10.1017/S174392131701047X. arXiv: 1802.05120 .

Silant'ev, N.A., Lekht, E.E., Mendoza-Torres, J.E., Rudnitskij, G.M., 2006. Influence of turbulence on the shape of a spectral line. The analytical approach. A\&A 453, 989-1002. https://doi.org/10.1051/ 0004-6361:20053069.

Sobolev, A.M., Wallin, B.K., Watson, W.D., 1998. Astrophysical maser radiation from a turbulent medium: application to $25 \mathrm{GHz}$ methanol masers. Astrophys. J. 498, 763-772. https://doi.org/10.1086/305575.

Sobolev, A.M., Watson, W.D., Okorokov, V.A., 2003. Images of astrophysical masers and their variability in a turbulent medium: the 25 GHz methanol masers. Astrophys. J. 590, 333-339. https://doi.org/ $10.1086 / 375002$.

Sobolev, A.M., Sutton, E.C., Cragg, D.M., Godfrey, P.D., 2005. Model of the W3(OH) Environment based on data for both maser and "QuasiThermal” methanol lines. AP\%SS 295, 189-196. doi: https://doi.org/ 10.1007/s10509-005-3686-x. arXiv:astro-ph/0409188.

Sobolev, A.M., Cragg, D.M., Ellingsen, S.P., Gaylard, M.J., Goedhart, S., Henkel, C., Kirsanova, M.S., Ostrovskii, A.B., Pankratova, N.V., Shelemei, O.V., van der Walt, D.J., Vasyunina, T.S., Voronkov, M.A., 2007. How do methanol masers manage to appear in the youngest star vicinities and isolated molecular clumps? In: Chapman, J.M., Baan, W. A. (Eds.), Astrophysical masers and their environments, volume 242 of IAU Symposium, Cambridge University Press, Cambridge, UK, 2007, pp. 81-88. doi: https://doi.org/10.1017/S1743921307012616. arXiv: 0706.3117.

Sobolev, A.M., Moran, J.M., Gray, M.D., Alakoz, A., Imai, H., Baan, W. A., Tolmachev, A.M., Samodurov, V.A., Ladeyshchikov, D.A., 2018. Sun-sized water vapor masers in cepheus A. Astrophys. J. 856, 60. https://doi.org/10.3847/1538-4357/aab096, arXiv:1802.06756.

Sobolev, A.M., Shakhvorostova, N.N., Alakoz, A.V., Baan, W.A., 2018. RadioAstron space-VLBI project: studies of masers in star forming regions of our Galaxy and megamasers in external galaxies. In: Tarchi, A., Reid, M.J., Castangia, P. (Eds.), Astrophysical Masers: Unlocking the Mysteries of the Universe, volume 336 of IAU Symposium, Cambridge University Press, Cambridge, UK, pp. 417-421. doi: https://doi.org/10.1017/S1743921317011401. arXiv:1802.05133.

Strelnitski, V., Alexander, J., Gezari, S., Holder, B.P., Moran, J.M., Reid, M.J., 2002. $\mathrm{H}_{2} \mathrm{O}$ masers and supersonic turbulence. Astrophys. J. 581, 1180-1193. https://doi.org/10.1086/344244 astro-ph/0210342.

Sugiyama, K., Fujisawa, K., Doi, A., Honma, M., Isono, Y., Kobayashi, H., Mochizuki, N., Murata, Y., 2008. A Synchronized Variation of the 6.7 GHz Methanol Maser in Cepheus A, PASJ 60, 1001-1006. doi: https://doi.org/10.1093/pasj/60.5.1001. arXiv: 0804.4623.

Szymczak, M., Olech, M., Wolak, P., Bartkiewicz, A., Gawroński, M., 2016. Discovery of periodic and alternating flares of the methanol and water masers in G107.298+5.639, MNRAS 459, L56-L60. doi: https://doi.org/10.1093/mnrasl/slw044. arXiv:1604.00796.

Szymczak, M., Olech, M., Sarniak, R., Wolak, P., Bartkiewicz, A., 2018. Monitoring observations of $6.7 \mathrm{GHz}$ methanol masers. MNRAS 474, 
219-253. doi:https://doi.org/10.1093/mnras/stx2693. arXiv:1710. 04595.

Takefuji, K., Imai, H., Sekido, M., 2016. Development of cross-correlation spectrometry and the coherent structures of maser sources. PASJ 68, 86. https://doi.org/10.1093/pasj/psw077, arXiv:1607.07938.

Vlemmings, W.H.T., van Langevelde, H.J., Diamond, P.J., 2005. The magnetic field around late-type stars revealed by the circumstellar $\mathrm{H}_{2} \mathrm{O}$ masers. A\&A 434, 1029-1038. doi: https://doi.org/10.1051/0004-6361: 20042488. arXiv: astro-ph/0501628.

Vlemmings, W.H.T., Khouri, T., Martí-Vidal, I., Tafoya, D., Baudry, A., Etoka, S., Humphreys, E.M.L., Jones, T.J., Kemball, A., O'Gorman, E., Pérez-Sánchez, A.F., Richards, A.M.S., 2017. Magnetically aligned dust and $\mathrm{SiO}$ maser polarisation in the envelope of the red supergiant VY Canis Majoris. A\&A 603, A92. https://doi.org/10.1051/0004-6361/ 201730735, arXiv:1703.02084.

Voronkov, M., Sobolev, A., Ellingsen, S., Ostrovskii, A., Alakoz, A., 2005. Maser action in methanol transitions. AP\&SS 295, 217-223. https://doi.org/10.1007/s10509-005-3692-z astro-ph/0407275.

Voronkov, M.A., Caswell, J.L., Ellingsen, S.P., Breen, S.L., Britton, T.R., Green, J.A., Sobolev, A.M., Walsh, A.J., 2012. New class I methanol masers. In: Booth, R.S., Vlemmings, W.H.T., Humphreys, E.M.L. (Eds.), Cosmic Masers - from OH to H0, volume 287 of IAU Symposium, Astronomical Society of the Pacific, San Francisco, USA, 2012, pp. 433-440. doi: https://doi.org/10.1017/S174392131200748X. arXiv: 1203.5492 .

Voronkov, M.A., Caswell, J.L., Ellingsen, S.P., Green, J.A., Breen, S.L., 2014. Southern class I methanol masers at 36 and $44 \mathrm{GHz}$. MNRAS 439, 2584-2617. https://doi.org/10.1093/mnras/stu116 1401.5179.

Wittkowski, M., Boboltz, D.A., Ohnaka, K., Driebe, T., Scholz, M., 2007. The Mira variable S Orionis: relationships between the photosphere, molecular layer, dust shell, and SiO maser shell at 4 epochs. A\&A 470, 191-210. https://doi.org/10.1051/0004-6361:20077168 0705.4614.

Yang, W., Xu, Y., Chen, X., Ellingsen, S.P., Lu, D., Ju, B., Li, Y., 2017. A New 95 GHz Methanol Maser Catalog. I. Data, ApJS 231, 20. doi: https://doi.org/10.3847/1538-4365/aa6ff3. arXiv: 1705.01806.

Zinchenko, I., Liu, S.-Y., Su, Y.-N., Sobolev, A.M., 2017. Detection of a new methanol maser line with ALMA. A\&A 606, L6. https://doi.org/ 10.1051/0004-6361/201731752, arXiv:1709.08889. 